\title{
Dissociative charmonia photoproduction as a signal of gluon saturation at LHC
}

\author{
Jan Cepila* \\ Czech Technical University in Prague \\ E-mail: jan.cepila@fjfi.cvut.cz \\ Jesús Guillermo Contreras Nuno \\ Czech Technical University in Prague \\ E-mail: jesus.guillermo.contreras.nunodcern.ch \\ Jesús Daniel Tapia Takaki \\ Department of Physics and Astronomy, The University of Kansas, Lawrence, KS, USA \\ E-mail: daniel.tapia.takaki@cern.ch
}

\begin{abstract}
We have developed a model in which we treat photoproduction of charmonia via the color dipole approach taking into account the quantum fluctuations of the proton structure. These fluctuations are characterized by hot spots, randomly generated in the transverse plane, whose number grows with decreasing Bjorken- $x$. Our model successfully reproduces the $F_{2}\left(x, Q^{2}\right)$ data from HERA at the relevant scale, as well as the exclusive and dissociative $J / \Psi$ photoproduction data from $\mathrm{H} 1$ and ALICE. Our model predicts that once the proton is fully populated by hot spots the dissociative $J / \Psi$ cross section reaches a maximum and then decreases steeply with energy. Our prediction provides a clear signature for gluon saturation at LHC energies.
\end{abstract}

25th International Workshop on Deep Inelastic Scattering and Related Topics

3-7 April 2017

University of Birmingham, Birmingham, $U K$

${ }^{*}$ Speaker. 


\section{Introduction}

Perturbative QCD predicts that the gluon density in hadrons grows with energy, or equivalently with decreasing Bjorken- $x$, up to a point where non-linear effects manifest to slow down this growth, a phenomenon known as gluon saturation; see e.g. [1].

Inclusive data on the proton structure function $F_{2}\left(x, Q^{2}\right)$ from deeply inelastic scattering at HERA [2] show that indeed the gluon density grows steeply at small $x$. At the same time the analysis of these data is inconclusive regarding the question of gluon saturation, because the data can be equally well described without, e.g. [2], or with, e.g. [3], the inclusion of saturation effects.

Exclusive vector meson photoproduction, see left panel of Fig 1, is sensitive to the distribution of gluons in the impact parameter plane, through the dependence of the cross section on $t$, the square of the momentum transfer at the target vertex.

In a Good-Walker formalism [4] exclusive diffractive processes are sensitive to the average over the different configurations of the target, while dissociative processes, where the target gets excited and produces many particles, measure the variance over the configurations [5].

In this contribution, we report on our studies [6], where we predicted the energy dependence of exclusive and dissociative $J / \Psi$ photoproduction off protons, showing that it provides a clear signature of gluon saturation at the LHC. Our calculation reproduces correctly the rise with $W_{\gamma_{p}}$ of this cross section as measured at HERA [7], and predicts that it reaches a maximum at $W_{\gamma p} \approx 500$ $\mathrm{GeV}$, followed by a steep decrease at higher energies. The LHC can explore a wide range of $W_{\gamma p}$, from a few tens of $\mathrm{GeV}$ to more than a $\mathrm{TeV}$, making it an ideal place to look for this clear signature of gluon saturation.

\section{The amplitude for photoproduction of vector mesons}

Diagrams for the exclusive and dissociative production of vector mesons are shown in Fig. 1 where the photon source is a lead ion. In the dipole colour model $[8,9]$ the photon fluctuates into a coherent quark-antiquark pair with the transverse distance $\vec{r}$, then this pair interacts with the target at impact parameter $\vec{b}$ with the colour dipole cross section $\sigma_{\text {dip }}$ and finally the dipole forms a vector meson. In this approach the amplitude can be written as follows (see for example [10]),

$$
A\left(x, Q^{2}, \vec{\Delta}\right)_{T, L}=i \int d \vec{r} \int_{0}^{1} \frac{d z}{4 \pi}\left(\Psi^{*} \Psi_{\mathrm{V}}\right)_{T, L}(r, z, Q) \int d \vec{b} e^{-i(\vec{b}-(1-z) \vec{r}) \cdot \vec{\Delta}} \frac{d \sigma_{\mathrm{dip}}}{d \vec{b}}
$$

where $\Psi_{\mathrm{V}}$ is the wave function of the vector meson formed from $q \bar{q}$ state, $\Psi$ is the wave function of a virtual photon fluctuating into a quark-antiquark colour dipole and $z$ is the fraction of the longitudinal momentum of the dipole carried by the quark. The subindices $T$ and $L$ refer to the contribution of the transversal and longitudinal degrees of freedom of the quasi-real photon, respectively. For $\Psi$ we use the definitions and parameter values of [11] and for $\Psi_{\mathrm{V}}$ we use the boosted gaussian model $[12,13]$, with the numerical values of the parameters as in [10].

The physics of the interaction is encoded in the dipole-target cross section, which is related, via the optical theorem, to the imaginary part of the forward dipole-target amplitude $N(x, \vec{r}, \vec{b})$ :

$$
\frac{d \sigma_{\mathrm{dip}}}{d \vec{b}}=2 N(x, \vec{r}, \vec{b})=\sigma_{0} N(x, r) T(\vec{b}) .
$$


We use a factorised form for the dipole amplitude (see [6]), where $\sigma_{0}$ is a constant that provides the normalisation and $T(\vec{b})$ describes the proton profile in the impact parameter plane. We chose the form of $N(x, r)$ given by the GBW model [11]

$$
N^{\mathrm{GBW}}(x, r)=\left(1-e^{-r^{2} Q_{s}^{2}(x) / 4}\right), \quad Q_{s}^{2}(x)=Q_{0}^{2}\left(x_{0} / x\right)^{\lambda}, \quad Q_{0}^{2}=1 \mathrm{GeV}^{2},
$$

where $\lambda$ and $x_{0}$ are free parameters.

In our model all fluctuations are included in the proton profile $T(\vec{b})$. Following $[6,14,15]$ we define the proton profile as the sum of $N_{h s}(x)$ regions of high gluon density, so called hot spots, each of them having a gaussian distribution:

$$
T(\vec{b})=\frac{1}{N_{h s}(x)} \sum_{i=1}^{N_{h s}(x)} T_{h s}\left(\vec{b}-\vec{b}_{i}\right), \quad T_{h s}\left(\vec{b}-\vec{b}_{i}\right)=\frac{1}{2 \pi B_{h s}} e^{-\frac{\left(\vec{b}-\vec{b}_{i}\right)^{2}}{2 B_{h s}}},
$$

where each $\vec{b}_{i}$ is obtained from a 2-dimensional gaussian distribution centred at $(0,0)$ and having a width $B_{p}$. The new ingredient in our model is that we introduce an indirect energy dependence of the proton profile $T(\vec{b})$ by making the number of hot spots grow with decreasing $x$. This implements the hypothesis that, at a given fixed scale, as energy increases, the number of gluons available for the interaction increases.

Using the amplitude (2.1), the cross sections are

$$
\frac{d \sigma(\gamma p \rightarrow J / \Psi p)}{d t}=\frac{R_{g}^{2}}{16 \pi}\left|\left\langle A\left(x, Q^{2}, \vec{\Delta}\right)\right\rangle\right|^{2}
$$

for the exclusive process, and

$$
\frac{d \sigma(\gamma p \rightarrow J / \Psi Y)}{d t}=\frac{R_{g}^{2}}{16 \pi}\left(\left\langle\left|A\left(x, Q^{2}, \vec{\Delta}\right)\right|^{2}\right\rangle-\left|\left\langle A\left(x, Q^{2}, \vec{\Delta}\right)\right\rangle\right|^{2}\right)
$$

for dissociative production, where $R_{g}$ is the so-called skewedness correction [16] given by

$$
R_{g}\left(\lambda_{g}\right)=\frac{2^{2 \lambda_{g}+3}}{\sqrt{\pi}} \frac{\Gamma\left(\lambda_{g}+5 / 2\right)}{\Gamma\left(\lambda_{g}+4\right)}, \quad \lambda_{g} \equiv \frac{\partial \ln \left(A_{L, T}\right)}{\partial \ln (1 / x)} .
$$

The final cross section is the sum of the $T$ and $L$ contributions.

\section{Results}

The parameters of the model were fixed as follows (see the discussion in [6]). The value of $\lambda$ is constrained by the energy dependence of exclusive $J / \Psi$ photoproduction to $\lambda=0.21$. The parameter $B_{p}$ is constrained by the $t$ distribution of the same data and set to $B_{p}=4.7 \mathrm{GeV}^{-2}$. We define $\sigma_{0} \equiv 4 \pi B_{p}$. The $x_{0}$ parameter is strongly correlated to $\sigma_{0}$, so once $B_{p}$ is fixed, the normalisation of the data yields $x_{0}=0.0002$. The parameter $B_{h s}$ is constrained by the $t$ dependence of the dissociative process and it is set to $B_{h s}=0.8 \mathrm{GeV}^{-2}$. Finally, as we relate the number of hot spots with the number of gluons available for the interaction, we parametrise

$$
N_{h s}(x)=p_{0} x^{p_{1}}\left(1+p_{2} \sqrt{x}\right)
$$



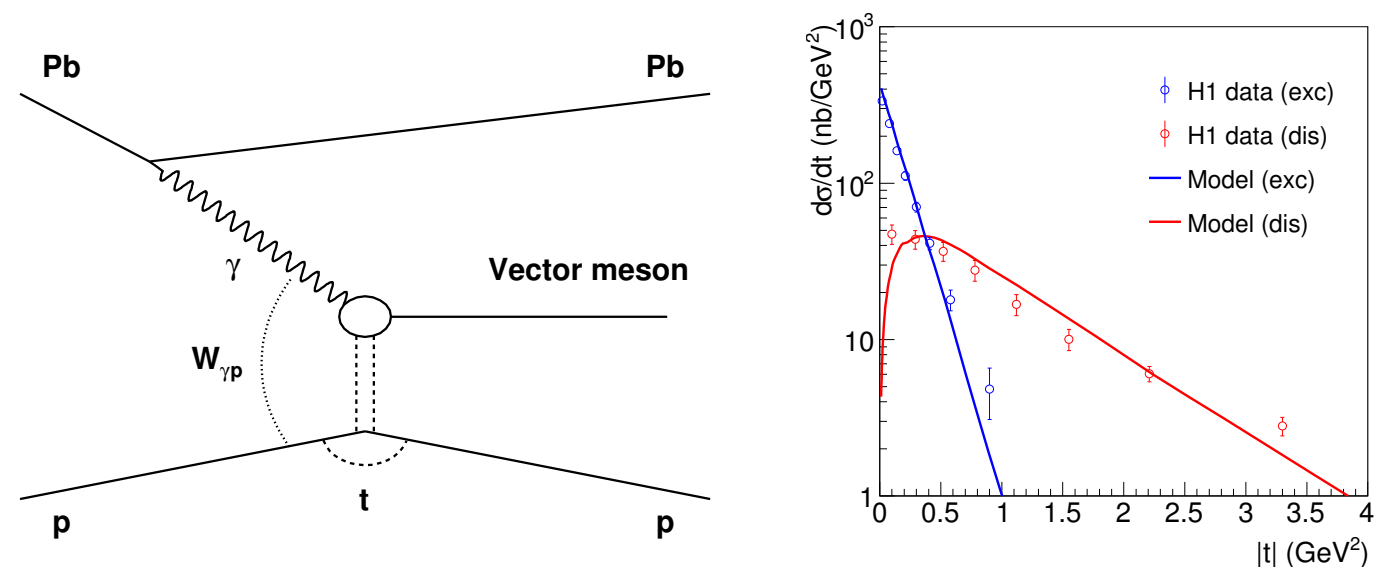

Figure 1: Left: Diagram for exclusive and dissociative vector meson photo-production. In the dissociative case the proton in the final state does not survive and produces a multi-particle final state Y. Right: Comparison of the model (solid lines) to data (open bullets) on the $|t|$ distribution of exclusive (blue) and dissociative (red) photoproduction of $J / \Psi$ as measured by $\mathrm{H} 1$ [7] at $\left\langle W_{\gamma p}\right\rangle=78 \mathrm{GeV}$ published in [6].

where we set $p_{0}=0.011, p_{1}=-0.58$ and $p_{2}=250$. Note that the parameters are chosen so that they describe simultaneously the energy dependence of $\mathrm{H} 1$ measurements of exclusive and dissociative $J / \Psi$ photoproduction.

Although the model is relatively simple and has been developed to describe vector meson photoproduction, it describes well the $F_{2}\left(x, Q^{2}\right)$ data (see [6]). The comparison with H1 data [7] on the $t$ dependence of $J / \Psi$ photoproduction is shown in the right panel of Fig. 1 for the data at $\left\langle W_{\gamma p}\right\rangle=78 \mathrm{GeV}$. Both the exclusive and the dissociative processes are reasonably well described. The left panel of Fig. 2 shows the energy dependence of the model and compares it to data from H1 [7] and ALICE [17]. Again, the model describes quite well the data.

Our main result is shown in the right panel of Fig. 2. We show that the model describes correctly the energy evolution of the dissociative cross section for the available data. We also show that the model predicts that the cross section will reach a maximum at $W_{\gamma p} \approx 500 \mathrm{GeV}$ and it will then turn around and decrease for higher energies. This decrease of the dissociative $J / \Psi$ photoproduction cross section is significantly large and can be observed in the $W_{\gamma p}$ energy range accessible at the LHC.

\section{Summary and discussion}

We have presented a model for the dissociative $J / \Psi$ photoproduction cross section. The model incorporates a fluctuating hot spot structure of the proton in the impact parameter plane. The new ingredient of the model is that the number of hot spots grows with decreasing $x$. The model describes correctly the behaviour of $F_{2}\left(x, Q^{2}\right)$ at the relevant scale, as well as the $W_{\gamma p}$ and $t$ dependence of the exclusive and dissociative $J / \Psi$ photoproduction cross section as measured by $\mathrm{H} 1$ and ALICE.

The energy dependence shown in the right panel of Fig. 2 has a geometrical origin reminiscent of percolation. At some point the number of hot spots is so large that they overlap. When the 

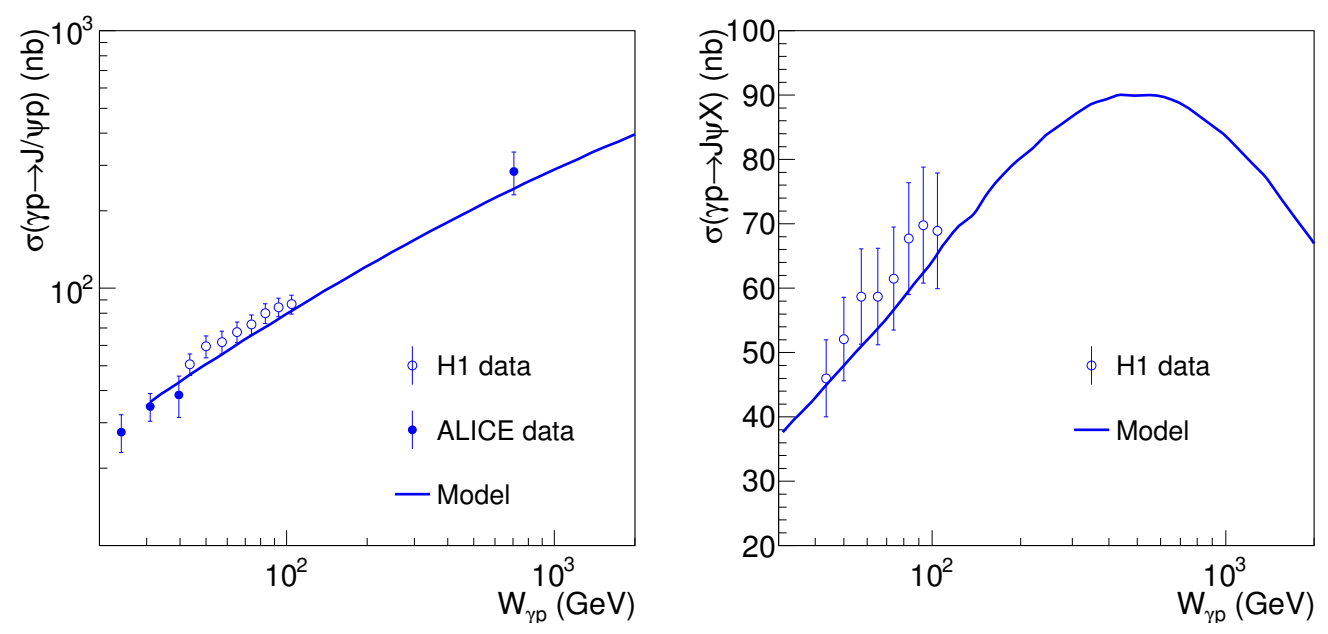

Figure 2: Comparison of the model (solid lines) to data on the $W_{\gamma p}$ dependence of the cross section for exclusive (left) and dissociative (right) photoproduction of $J / \Psi$ as measured by H1 [7] and ALICE [17] (open and solid bullets, respectively).

overlap is large enough, different configurations look the same and the variance decreases. The model predicts that the energy dependence of the dissociative process increases from low energies up to a maximum and then decreases steeply. For the parameters that we have employed in our study, the maximum at $W_{\gamma p} \approx 500 \mathrm{GeV}$ is reached when some 10 hot spots are present, indicating a sizeable overlap of hot spots. This behaviour happens within the energy range accessible at the LHC.

In our model the shape of the $t$ dependence of the cross sections for the dissociative and exclusive processes does not vary with $x$. However, if the dissociative $J / \Psi$ cross section disappears at relatively high energies as predicted in our model, it will simplify subtracting the dissociative background in the measurement of the $t$ distribution of exclusive vector meson photoproduction, particularly at large $|t|$ values. This is important since the appearance of diffractive dips in the $t$ distribution at large $|t|$ has been proposed as a good observable for gluon saturation effects [18, 19].

\section{Acknowledgments}

This work was supported by the grant 17-04505S of the Czech Science Foundation.

\section{References}

[1] F. Gelis, E. Iancu, J. Jalilian-Marian and R. Venugopalan, The Color Glass Condensate, Ann. Rev. Nucl. Part. Sci. 60 (2010) 463-489, [1 002 . 0333].

[2] ZEUS, H1 collaboration, H. Abramowicz et al., Combination of measurements of inclusive deep inelastic $e^{ \pm} p$ scattering cross sections and QCD analysis of HERA data, Eur. Phys. J. $\mathbf{C 7 5}$ (2015) 580, [1506.06042].

[3] J. L. Albacete, N. Armesto, J. G. Milhano, P. Quiroga-Arias and C. A. Salgado, AAMQS: A non-linear QCD analysis of new HERA data at small-x including heavy quarks, Eur.Phys.J. C71 (2011) 1705, [1012.4408]. 
[4] M. L. Good and W. D. Walker, Diffraction disssociation of beam particles, Phys. Rev. 120 (1960) $1857-1860$.

[5] H. I. Miettinen and J. Pumplin, Diffraction Scattering and the Parton Structure of Hadrons, Phys. Rev. D18 (1978) 1696.

[6] J. Cepila, J. G. Contreras and J. D. Tapia Takaki, Energy dependence of dissociative J/ $\psi$ photoproduction as a signature of gluon saturation at the LHC, Phys. Lett. B766 (2017) 186-191, [1608.07559].

[7] H1 collaboration, C. Alexa et al., Elastic and Proton-Dissociative Photoproduction of J/psi Mesons at HERA, Eur. Phys. J. C73 (2013) 2466, [1304 . 5162].

[8] A. H. Mueller, Small x Behavior and Parton Saturation: A QCD Model, Nucl. Phys. B335 (1990) 115-137.

[9] N. N. Nikolaev and B. G. Zakharov, Color transparency and scaling properties of nuclear shadowing in deep inelastic scattering, Z. Phys. C49 (1991) 607-618.

[10] H. Kowalski, L. Motyka and G. Watt, Exclusive diffractive processes at HERA within the dipole picture, Phys.Rev. D74 (2006) 074016, [hep-ph / 0606272$].$

[11] K. J. Golec-Biernat and M. Wusthoff, Saturation effects in deep inelastic scattering at low $Q^{2}$ and its implications on diffraction, Phys.Rev. D59 (1998) 014017, [hep-ph/ 9807513$].$

[12] J. Nemchik, N. N. Nikolaev and B. Zakharov, Scanning the BFKL pomeron in elastic production of vector mesons at HERA, Phys.Lett. B341 (1994) 228-237, [hep-ph/9405355].

[13] J. Nemchik, N. N. Nikolaev, E. Predazzi and B. Zakharov, Color dipole phenomenology of diffractive electroproduction of light vector mesons at HERA, Z.Phys. C75 (1997) 71-87, [hep-ph / 9605231 ].

[14] H. Mäntysaari and B. Schenke, Evidence of strong proton shape fluctuations from incoherent diffraction, Phys. Rev. Lett. 117 (2016) 052301, [1603.04349].

[15] H. Mäntysaari and B. Schenke, Revealing proton shape fluctuations with incoherent diffraction at high energy, Phys. Rev. D94 (2016) 034042, [1607.01711].

[16] A. G. Shuvaev, K. J. Golec-Biernat, A. D. Martin and M. G. Ryskin, Off diagonal distributions fixed by diagonal partons at small $x$ and xi, Phys. Rev. D60 (1999) 014015, [hep-ph/9902410].

[17] ALICE collaboration, B. B. Abelev et al., Exclusive J/ $\psi$ photoproduction off protons in ultra-peripheral p-Pb collisions at $\sqrt{s_{\mathrm{NN}}}=5.02$ TeV, Phys. Rev. Lett. 113 (2014) 232504, [1406.7819].

[18] T. Toll and T. Ullrich, Exclusive diffractive processes in electron-ion collisions, Phys. Rev. C87 (2013) 024913, [1211.3048].

[19] N. Armesto and A. H. Rezaeian, Exclusive vector meson production at high energies and gluon saturation, Phys.Rev. D90 (2014) 054003, [1 402 . 4831]. 